\title{
The topology of restricted partition posets
}

\author{
Richard Ehrenborg ${ }^{1 /}$ and JiYoon Jung ${ }^{1}$ \\ ${ }^{1}$ Department of Mathematics, University of Kentucky, Lexington, KY, USA
}

\begin{abstract}
For each composition $\vec{c}$ we show that the order complex of the poset of pointed set partitions $\Pi_{\vec{c}}^{\bullet}$ is a wedge of $\beta(\vec{c})$ spheres of the same dimensions, where $\beta(\vec{c})$ is the number of permutations with descent composition $\vec{c}$. Furthermore, the action of the symmetric group on the top homology is isomorphic to the Specht module $S^{B}$ where $B$ is a border strip associated to the composition $\vec{c}$. We also study the filter of pointed set partitions generated by a knapsack integer partitions and show the analogous results on homotopy type and action on the top homology.
\end{abstract}

Résumé. Pour chaque composition $\vec{c}$ nous montrons que le complexe simplicial des chaînes de l'ensemble ordonné $\Pi_{\vec{c}}^{\bullet}$ des partitions pointées d'un ensemble est un bouquet de $\beta(\vec{c})$ sphères de même dimension, où $\beta(\vec{c})$ est le nombre de permutations ayant la composition de descentes $\vec{c}$. De plus, l'action du groupe symétrique sur le groupe d'homologie de degré maximum est isomorphe au module de Specht $S^{B}$ où $B$ est la bande frontalière associée à la composition $\vec{c}$. Nous étudions aussi le filtre des partitions pointées d'un ensemble, engendré par des partitions d'entiers de type "sac à dos" et nous démontrons des résultats analogues pour le type d'homotopie et pour l'action sur le groupe d'homologie de degré maximum.

Keywords: Pointed set partitions, descent set statistics, top homology group, Specht module, knapsack partitions.

\section{Introduction}

The study of partitions with restrictions on their block sizes began in the dissertation by Sylvester [16], who studied the poset $\Pi_{n}^{2}$ of partitions where every block has even size. He proved that the Möbius function of this poset is given by $\mu\left(\Pi_{n}^{2} \cup\{\hat{0}\}\right)=(-1)^{n / 2} \cdot E_{n-1}$, where $E_{n}$ denotes the $n$th Euler number. Recall that the $n$th Euler number enumerates the number of alternating permutations, that is, permutations $\alpha=\alpha_{1} \cdots \alpha_{n}$ in the symmetric group $\mathfrak{S}_{n}$ such that $\alpha_{1}<\alpha_{2}>\alpha_{3}<\alpha_{4}>\cdots$. Stanley [13] generalized this result to the $d$-divisible partition lattice $\Pi_{n}^{d}$, that is, the collection of partitions of $\{1,2, \ldots, n\}$ where each block size is divisible by $d$. His results states that the Möbius function $\mu\left(\Pi_{n}^{d} \cup\{\hat{0}\}\right)$ is, up to the $\operatorname{sign}(-1)^{n / d}$, the number of permutations in $\mathfrak{S}_{n-1}$ with descent set $\{d, 2 d, \ldots, n-d\}$, in other words, the number of permutations with descent composition $(d, \ldots, d, d-1)$.

Calderbank, Hanlon and Robinson [3] extended these results by considering the action of the symmetric group $\mathfrak{S}_{n-1}$ on the top homology group of the order complex of $\Pi_{n}^{d}-\{\hat{1}\}$. They showed this action is the Specht module on the border strip corresponding the composition $(d, \ldots, d, d-1)$.

\footnotetext{
${ }^{\dagger}$ Both authors are partially supported by National Science Foundation grants 0902063. 
Wachs [17] showed that the $d$-divisible partition lattice has an $E L$-shelling, and hence as a corollary obtained that the homotopy type is a wedge of spheres of dimension $n / d-2$. She then gave a more explicit proof of the representation of the top homology of $\Pi_{n}^{d}-\{\hat{1}\}$.

So far we note that the $d$-divisible partition lattice is closely connected with the permutations having the descent composition $(d, \ldots, d, d-1)$. We explain this phenomenon in this paper by introducing pointed partitions. They are partitions where one block is considered special, called the pointed block. We obtain such a partition by removing the element $n$ from its block and making this block the pointed block. We now extend the family of posets under consideration. For each composition $\vec{c}=\left(c_{1}, \ldots, c_{k}\right)$ of $n$ we define a poset $\Pi_{\vec{c}}^{\bullet}$ such that the Möbius function $\mu\left(\Pi_{\vec{c}}^{\bullet} \cup\{\hat{0}\}\right)$ is the sign $(-1)^{k}$ times the number of permutations with descent composition $\vec{c}$. Furthermore, the order complex of $\Pi_{\vec{c}}^{\bullet}-\{\hat{1}\}$ is homotopy equivalent to a wedge of spheres of dimension $k-2$. Finally, we show the action of the symmetric group on the top homology group $\widetilde{H}_{k-2}\left(\Delta\left(\Pi_{\vec{c}}^{\bullet}-\{\hat{1}\}\right)\right)$ is the Specht module corresponding to the composition $\vec{c}$.

Our techniques differ from Wachs' method for studying the $d$-divisible partition lattice. Using Quillen's fiber lemma, we are able to change the poset question into studying the subcomplexes of the complex of ordered partitions. These subcomplexes are in fact order complexes of rank-selected Boolean algebras and hence shellable. The homotopy equivalence given by Quillen's fiber lemma also carries the action of the symmetric group.

Ehrenborg and Readdy [4] studied the Möbius function of filters of the partition lattice. They defined the notion of a knapsack partition. For a filter of a knapsack partition they showed that its Möbius function was a sum of descent set statistics. We now extend these results topologically by showing that the associated order complex is a wedge of spheres. The proof follows the same outline as the previous study except that we use discrete Morse theory to determine the homotopy type of the associated complexes of ordered set partitions. Furthermore we obtain that the action of the symmetric group on the the top homology is a direct sum of Specht modules.

We end the paper with open questions for future research.

\section{Preliminaries}

For basic notions concerning partially ordered sets (posets), see the book by Stanley [14]. For topological background, see the article by Björner [2] and the book by Kozlov [10]. Finally, for representation theory for the symmetric group, see Sagan [12].

Let $[n]$ denote the set $\{1,2, \ldots, n\}$ and for $i \leq j$ let $[i, j]$ denote the interval $\{i, i+1, \ldots, j\}$. A pointed set partition $\pi$ of the set $[n]$ is a pair $(\sigma, Z)$, where $Z$ is a subset of $[n]$ and $\sigma=\left\{B_{1}, B_{2}, \ldots, B_{k}\right\}$ is a partition of the set difference $[n]-Z$. We will write the pointed partition $\pi$ as

$$
\pi=\left\{B_{1}, B_{2}, \ldots, B_{k}, \underline{Z}\right\},
$$

where we underline the set $Z$ and we write $1358|4| \underline{267}$ as shorthand for $\{\{1,3,5,8\},\{4\},\{2,6,7\}\}$. Moreover, we call the set $Z$ the pointed block. Let $\Pi_{n}^{\bullet}$ denote the set of all pointed set partitions on the set $[n]$. The set $\Pi_{n}^{\bullet}$ has a natural poset structure. The order relation is given by

$$
\begin{aligned}
& \left\{B_{1}, B_{2}, \ldots, B_{k}, \underline{Z}\right\}<\left\{B_{1} \cup B_{2}, \ldots, B_{k}, \underline{Z}\right\}, \\
& \left\{B_{1}, B_{2}, \ldots, B_{k}, \underline{Z}\right\}<\left\{B_{2}, \ldots, B_{k}, \underline{B_{1} \cup Z}\right\} .
\end{aligned}
$$

The lattice $\Pi_{n}^{\bullet}$ is isomorphic to the partition lattice $\Pi_{n+1}$ by the bijection $\left\{B_{1}, \ldots, B_{k}, \underline{Z}\right\} \longmapsto$ $\left\{B_{1}, \ldots, B_{k}, Z \cup\{n+1\}\right\}$. However it is to our advantage to work with pointed set partitions. 
For a permutation $\alpha=\alpha_{1} \cdots \alpha_{n}$ in the symmetric group $\mathfrak{S}_{n}$ define its descent set to be the set

$$
\left\{i \in[n-1]: \alpha_{i}>\alpha_{i+1}\right\} .
$$

Subsets of $[n-1]$ are in a natural bijective correspondence with compositions of $n$. Hence we define the descent composition of the permutation $\alpha$ to be the composition

$$
\operatorname{Des}(\alpha)=\left(s_{1}, s_{2}-s_{1}, s_{3}-s_{2}, \ldots, s_{k-1}-s_{k-2}, n-s_{k-1}\right)
$$

where the descent set of $\alpha$ is the set $\left\{s_{1}<s_{2}<\cdots<s_{k-1}\right\}$. We define an integer composition $\vec{c}=\left(c_{1}, \ldots, c_{k}\right)$ to be a list of positive integers $c_{1}, \ldots, c_{k-1}$ and a non-negative integer $c_{k}$ with $c_{1}+$ $\cdots+c_{k}=n$. Note that the only part allowed to be 0 is the last part. Let $\beta(\vec{c})$ denote the number of permutations in $\alpha \in \mathfrak{S}_{n}$ with descent composition $\vec{c}$ for $c_{k}>0$. If $c_{k}=0$, let $\beta(\vec{c})=0$ for $k \geq 2$ and $\beta(\vec{c})=1$ for $k=1$.

On the set of compositions on $n$ we define an order relation by letting the cover relation be adding adjacent entries, that is,

$$
\left(c_{1}, \ldots, c_{i}, c_{i+1}, \ldots, c_{k}\right) \prec\left(c_{1}, \ldots, c_{i}+c_{i+1}, \ldots, c_{k}\right) .
$$

Observe that this poset is isomorphic to the Boolean algebra $B_{n}$ on $n$ elements and the maximal and minimal elements are the two compositions $(n)$ and $(1, \ldots, 1,0)$.

An integer partition $\lambda$ of a non-negative integer $n$ is a multiset of positive integers whose sum is $n$. We will indicate multiplicities with a superscript. Thus $\{5,3,3,2,1,1,1\}=\left\{5,3^{2}, 2,1^{3}\right\}$ is a partition of 16 . A pointed integer partition $(\lambda, m)$ of $n$ is pair where $m$ is a non-negative integer and $\lambda$ is a partition of $n-m$. We write this as $\left\{\lambda_{1}, \ldots, \lambda_{p}, \underline{m}\right\}$ where $\lambda=\left\{\lambda_{1}, \ldots, \lambda_{p}\right\}$ is the partition and $m$ is the pointed part. This notion of pointed integer partition is related to pointed set partitions by defining the type of a pointed set partition $\pi=\left\{B_{1}, B_{2}, \ldots, B_{k}, \underline{Z}\right\}$ to be the pointed integer partition

$$
\operatorname{type}(\pi)=\left\{\left|B_{1}\right|,\left|B_{2}\right|, \ldots,\left|B_{k}\right|, \underline{|Z|}\right\} .
$$

Similarly, the type of a composition $\vec{c}=\left(c_{1}, \ldots, c_{k}\right)$ is the pointed integer partition

$$
\operatorname{type}(\vec{c})=\left\{c_{1}, \ldots, c_{k-1}, \underline{c_{k}}\right\} \text {. }
$$

We now define the poset central to this paper.

Definition 2.1 For $\vec{c}$ a composition of $n$, let $\Pi_{\vec{c}}^{\bullet}$ be the subposet of the pointed partition lattice $\Pi_{n}^{\bullet}$ described by

$$
\Pi_{\vec{c}}^{\bullet}=\left\{\pi \in \Pi_{n}^{\bullet}: \exists \vec{d} \geq \vec{c}, \operatorname{type}(\pi)=\operatorname{type}(\vec{d})\right\} .
$$

In other words, the poset $\Pi_{\vec{c}}^{\bullet}$ consists of all pointed set partitions such that their type is the type of some composition $\vec{d}$ which is greater or equal to the composition $\vec{c}$ in the composition order.

Example 2.2 Consider the composition $\vec{c}=(d, \ldots, d, d-1)$ of the integer $n=d \cdot k-1$. For a composition to be greater than or equal to $\vec{c}$, it is equivalent to all its parts must be divisible by $d$ except the last part which is congruent to $d-1$ modulo $d$. Hence $\Pi_{\vec{c}}^{\bullet}$ consists of all pointed set partitions where the block sizes are divisible by $\vec{d}$ except the pointed block whose size is congruent to $d-1$ modulo $d$. Hence the poset $\Pi_{\vec{c}}^{\bullet}$ is isomorphic to the $d$-divisible partition lattice $\Pi_{n+1}^{d}$. 
Example 2.3 We note that $\Pi_{\vec{c}}^{\bullet} \cup\{\hat{0}\}$ is in general not a lattice. Consider the composition $\vec{c}=(1,1,2,1)$ and the four pointed set partitions

$$
\pi_{1}=1|2| 34\left|\underline{5}, \quad \pi_{2}=2\right| 5|34| \underline{1}, \quad \pi_{3}=34 \mid \underline{125} \text { and } \pi_{4}=2 \mid \underline{1345}
$$

in $\Pi_{(1,1,2,1)}^{\bullet}$. In the pointed partition lattice $\Pi_{5}^{\bullet}$ we have that $\pi_{1}, \pi_{2}<2|34| \underline{15}<\pi_{3}, \pi_{4}$. Since the pointed set partition $2|34| \underline{15}$ does not belong to $\Pi_{(1,1,2,1)}^{\bullet}$, we conclude that $\Pi_{(1,1,2,1)}^{\bullet} \cup\{\hat{0}\}$ is not a lattice.

For a poset $P$ define its order complex to be the simplicial complex $\Delta(P)$ where the vertices of the complex $\Delta(P)$ are the elements of the poset $P$ and the faces are the chains in poset. In other words, the order complex of $P$ is given by

$$
\Delta(P)=\left\{\left\{x_{1}, x_{2}, \ldots, x_{k}\right\}: x_{1}<x_{2}<\cdots<x_{k}, x_{1}, \ldots, x_{k} \in P\right\} .
$$

For the remainder of this section, we restrict ourselves to considering compositions of $n$ where the last part is positive. Such a composition lies in the interval from $(1, \ldots, 1)$ to $(n)$. This interval is isomorphic to the Boolean algebra $B_{n-1}$ which is a complemented lattice. Hence for such a composition $\vec{c}$ there exists a complementary composition $\vec{c}^{\prime}$ such that $\vec{c} \wedge \vec{c}^{\prime}=(1, \ldots, 1)$ and $\vec{c} \vee \vec{c}^{\prime}=(n)$. As an example, the complement of the composition $(1,3,1,1,4)=(1,1+1+1,1,1,1+1+1+1)$ is obtained by exchanging commas and plus signs, that is, $(1+1,1,1+1+1+1,1,1,1)=(2,1,4,1,1,1)$. Note that the complementary composition has $n-k+1$ parts.

For a composition $\vec{c}=\left(c_{1}, \ldots, c_{k}\right)$ define the intervals $R_{1}, \ldots, R_{k}$ by $R_{i}=\left[c_{1}+\cdots+c_{i-1}+\right.$ $\left.1, c_{1}+\cdots+c_{i}\right]$. Define the subgroup $\mathfrak{S}_{\vec{c}}$ of the symmetric group $\mathfrak{S}_{n}$ by $\mathfrak{S}_{\vec{c}}=\mathfrak{S}_{R_{1}} \times \cdots \times \mathfrak{S}_{R_{k}}$. Let $K_{1}, \ldots, K_{n-k+1}$ be the corresponding intervals for the complementary composition $\vec{c}^{\prime}$. Define the subgroup $\mathfrak{S}_{\vec{c}}^{\prime}$ by

$$
\mathfrak{S}_{\vec{c}}^{\prime}=\mathfrak{S}_{\vec{c}^{\prime}}=\mathfrak{S}_{K_{1}} \times \cdots \times \mathfrak{S}_{K_{n-k+1}} .
$$

A border strip is a connected skew shape which does not contain a 2 by 2 square [15, Section 7.17]. For each composition $\vec{c}$ there is a unique border strip $B$ that has $k$ rows and the $i$ th row from below consists of $c_{i}$ boxes. If we label the $n$ boxes of the border strip from southwest to northeast, then the intervals $R_{1}, \ldots, R_{k}$ correspond to the rows and the intervals $K_{1}, \ldots, K_{n-k+1}$ correspond to the columns. Furthermore, the group $\mathfrak{S}_{\vec{c}}$ is the row stabilizer and the group $\mathfrak{S}_{\vec{c}}^{\prime}$ is the column stabilizer of the border strip $B$.

\section{The simplicial complex of ordered set partitions}

An ordered set partition $\tau$ of set $S$ is a list of blocks $\left(C_{1}, C_{2}, \ldots, C_{m}\right)$ where the blocks are subsets of the set $S$ satisfying:

(i) All blocks except possibly the last block are non-empty, that is, $C_{i} \neq \emptyset$ for $1 \leq i \leq m-1$.

(ii) The blocks are pairwise disjoint, that is, $C_{i} \cap C_{j}=\emptyset$ for $1 \leq i<j \leq m$.

(iii) The union of the blocks is $S$, that is, $C_{1} \cup \cdots \cup C_{m}=S$.

To distinguish from pointed partitions we write $36-127-8-45$ for $(\{3,6\},\{1,2,7\},\{8\},\{4,5\})$. The type of an ordered set partitions, type $(\tau)$, is the composition $\left(\left|C_{1}\right|,\left|C_{2}\right|, \ldots,\left|C_{m}\right|\right)$. 
Let $\Delta_{n}$ denote the collection of all ordered set partitions of the set $[n]$. We view $\Delta_{n}$ as a simplicial complex. The ordered set partition $\tau=\left(C_{1}, C_{2}, \ldots, C_{m}\right)$ is an $(m-2)$-dimensional face. It has $m-1$ facets, which are $\tau=\left(C_{1}, \ldots, C_{i} \cup C_{i+1}, \ldots, C_{m}\right)$ for $1 \leq i \leq m-1$. The empty face corresponds to the ordered partition $([n])$. The complex $\Delta_{n}$ has $2^{n}-1$ vertices that are of the form $\left(C_{1}, C_{2}\right)$ where $C_{1} \neq \emptyset$. Moreover there are $n$ ! facets corresponding to permutations in the symmetric group $\mathfrak{S}_{n}$, that is, for a permutation $\alpha=\alpha_{1} \cdots \alpha_{n}$, the associated facet is $\left(\left\{\alpha_{1}\right\},\left\{\alpha_{2}\right\}, \ldots,\left\{\alpha_{n}\right\}, \emptyset\right)$.

The permutahedron is the $(n-1)$-dimensional polytope obtained by taking the convex hull of the $n$ ! points $\left(\alpha_{1}, \ldots, \alpha_{n}\right)$ where $\alpha=\alpha_{1} \cdots \alpha_{n}$ ranges over all permutations in the symmetric group $\mathfrak{S}_{n}$. Let $P_{n}$ denote the boundary complex of the dual of the $(n-1)$-dimensional permutahedron. Since the permutahedron is a simple polytope the complex $P_{n}$ is a simplicial complex homeomorphic to an $(n-2)$ dimensional sphere. In fact, it is the boundary of the complex $\Delta_{n}$.

For a permutation $\alpha=\alpha_{1} \cdots \alpha_{n}$ in the symmetric group $\mathfrak{S}_{n}$ and a composition $\vec{c}=\left(c_{1}, \ldots, c_{k}\right)$ of $n$, define the ordered partition

$$
\begin{aligned}
\sigma(\alpha, \vec{c}) & =\left(\left\{\alpha_{j}: j \in R_{i}\right\}\right)_{1 \leq i \leq k} \\
& =\left(\left\{\alpha_{1}, \ldots, \alpha_{c_{1}}\right\},\left\{\alpha_{c_{1}+1}, \ldots, \alpha_{c_{1}+c_{2}}\right\}, \ldots,\left\{\alpha_{c_{1}+\cdots+c_{k-1}+1}, \ldots, \alpha_{n}\right\}\right) .
\end{aligned}
$$

We write $\sigma(\alpha)$ when it is clear from the context what the composition $\vec{c}$ is.

For a composition $\vec{c}$ define the subcomplex $\Delta_{\vec{c}}$ to be

$$
\Delta_{\vec{c}}=\left\{\tau \in \Delta_{n}: \vec{c} \leq \operatorname{type}(\tau)\right\} .
$$

This complex has all of its facets of type $\vec{c}$. Especially, each facet has the form $\sigma(\alpha, \vec{c})$ for some permutation $\alpha$. As an example, note that $\Delta_{(1,1, \ldots, 1)}$ is the complex $P_{n}$.

However for a facet $F$ in $\Delta_{\vec{c}}$ there are $\vec{c} !=c_{1} ! \cdots c_{k}$ ! permutations that map to it by the function $\sigma$. Let $\sigma^{-1}(F)$ denote the unique permutation $\alpha$ that gets mapped to the facet $F$ which satisfies the inequalities

$$
\alpha_{c_{1}+\cdots+c_{i}+1}<\cdots<\alpha_{c_{1}+\cdots+c_{i+1}}
$$

for $0 \leq i \leq k-1$. Furthermore, the descent composition of the permutation $\sigma^{-1}(F)$ is greater than or equal to the composition $\vec{c}$, that is, $\operatorname{Des}\left(\sigma^{-1}(F)\right) \geq \vec{c}$.

Lemma 3.1 If the composition $\vec{c}=\left(c_{1}, \ldots, c_{k}\right)$ ends with 0 , then the simplicial complex $\Delta_{\vec{c}}$ is a cone over the complex $\Delta_{\left(c_{1}, \ldots, c_{k-1}\right)}$ with apex $([n], \emptyset)$ and hence contractible.

Theorem 3.2 Let $\vec{c}$ be a composition not ending with a zero. Then the simplicial complex $\Delta_{\vec{c}}$ is shellable. The spanning facets (also known as the homology facets) are of the form $\sigma(\alpha)$ where $\alpha$ ranges over all permutations in the symmetric group $\mathfrak{S}_{n}$ with descent composition $\vec{c}$, that is, $\operatorname{Des}(\alpha)=\vec{c}$. Hence the complex $\Delta_{\vec{c}}$ is homotopy equivalent to wedge of $\beta(\vec{c})$ spheres of dimension $k-2$.

By observing that $\Delta_{\vec{c}}$ is the order complex of a rank selection of the Boolean algebra $B_{n}$, this result is a direct consequence of that $B_{n}$ is $E L$-shellable [1].

\section{The homotopy type of the poset $\Pi_{\vec{c}}^{\bullet}$}

We now will use Quillen's fiber lemma to show that the chain complex $\Delta\left(\Pi_{\vec{c}}^{\bullet}-\{\hat{1}\}\right)$ is homotopy equivalent to the simplicial complex $\Delta_{\vec{c}}$. Recall that a simplicial map $f$ from a simplicial complex $\Gamma$ to a 


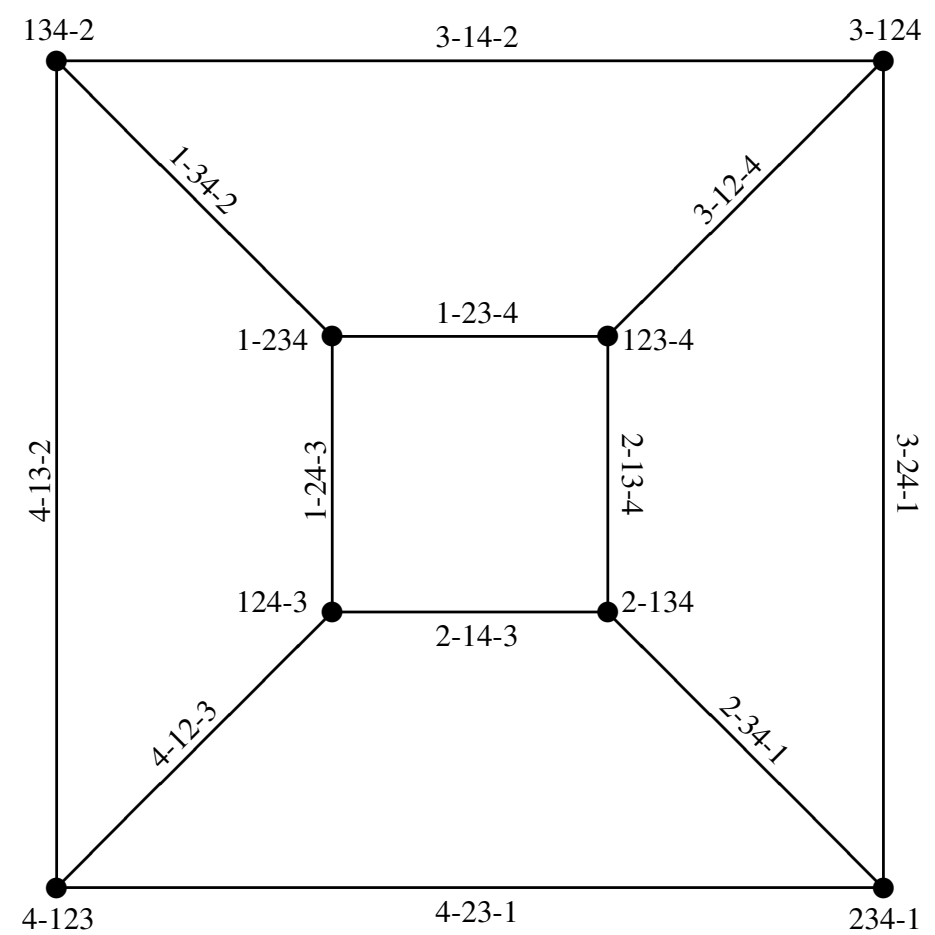

Fig. 1: The simplicial complex $\Delta_{(1,2,1)}$ of ordered partitions. Note that the ordered partition 1234 corresponds to the empty face.

poset $P$ sends vertices of $\Gamma$ to elements of $P$ and faces of the simplicial complex to chains of $P$. We have the following result due to Quillen [11].

Theorem 4.1 (Quillen's Fiber Lemma) Let $f$ be a simplicial map from the simplicial complex $\Gamma$ to the poset $P$ such that for all $x$ in $P$, the subcomplex $\Delta\left(f^{-1}\left(P_{\geq x}\right)\right)$ is contractible. Then the order complex $\Delta(P)$ and the simplicial complex $\Gamma$ are homotopy equivalent.

Quillen's proof of this result uses homotopy colimits. The proof of this result due to Walker [19] shows that the continuous function $f:|\Gamma| \longrightarrow|\Delta(P)|$ has a homotopy inverse. We will use this later when we study the action of the symmetric group in Section 6 .

Recall that the barycentric subdivision of a simplicial complex $\Gamma$ is the simplicial complex $\operatorname{sd}(\Gamma)$ whose vertices are the non-empty faces of $\Gamma$ and faces are subsets of chains of faces in $\Gamma$ ordered by inclusion. It is well-known that $\Gamma$ and $\operatorname{sd}(\Gamma)$ are homeomorphic (since they have the same geometric realization) and hence are homotopy equivalent.

Consider the map $\phi$ that sends an ordered set partition $\left(C_{1}, C_{2}, \ldots, C_{k}\right)$ to the pointed partition $\left\{C_{1}\right.$, $C_{2}, \ldots, C_{k-1}, \underline{C_{k}}$. We call this map the forgetful map since it forgets about the order between the blocks except it keeps the last part as the pointed block. Observe that the inverse image of the pointed partition $\left\{C_{1}, C_{2}, \ldots, C_{k-1}, C_{k}\right\}$ consists of $(k-1)$ ! ordered set partitions.

Lemma 4.2 Let $\pi$ be the pointed partition $\left\{B_{1}, \ldots, B_{m-1}, \underline{B_{m}}\right\}$ where $m \geq 2$. Let $\Omega$ be the subcomplex 
of the complex $\Delta_{\vec{c}}$ whose facets are given by the inverse image

$$
\phi^{-1}\left(\left(\Pi_{\vec{c}}^{\bullet}-\{\hat{1}\}\right)_{\geq \pi}\right)
$$

Then the complex $\Omega$ is a cone over the apex $\left([n]-B_{m}, B_{m}\right)$ and hence is contractible.

Theorem 4.3 The order complex $\Delta\left(\Pi_{\vec{c}}^{\bullet}-\{\hat{1}\}\right)$ is homotopy equivalent to the barycentric subdivision $\operatorname{sd}\left(\Delta_{\vec{c}}\right)$ and hence $\Delta_{\vec{c}}$.

By considering the reduced Euler characteristic of the complex $\Delta\left(\Pi_{\vec{c}}^{\bullet}-\{\hat{1}\}\right)$, we have the following corollary.

Corollary 4.4 The Möbius function of the poset $\Pi_{\vec{c}}^{\bullet} \cup\{\hat{0}\}$ is given by $(-1)^{k} \cdot \beta(\vec{c})$.

We note that this corollary can be given a combinatorial proof which avoids Quillen's fiber lemma.

\section{Cycles in the complex $\Delta_{\vec{c}}$}

In this section and the next we assume that the last part of the composition $\vec{c}$ is non-zero, since in the case $c_{k}=0$ the homology group is the trivial group; see Lemma 3.1 .

For $\alpha$ a permutation in the symmetric group $\mathfrak{S}_{n}$, define the subcomplex $\Sigma_{\alpha}$ of the complex $\Delta_{\vec{c}}$ to be the simplicial complex whose facets are given by $\left\{\sigma(\alpha \circ \gamma): \gamma \in \mathfrak{S}_{\vec{c}}^{\prime}\right\}$.

Lemma 5.1 The subcomplex $\Sigma_{\alpha}$ is isomorphic to the join of the duals of the permutahedra $P_{\left|K_{1}\right|} * \cdots *$ $P_{\left|K_{n-k+1}\right|}$ and hence it is sphere of dimension $k-2$.

Observe that the facets of $\Delta_{\vec{c}}$ are in bijection with permutations $\alpha$ such that $\operatorname{Des}(\alpha) \geq \vec{c}$ in the composition order.

Recall that the boundary map of the face $\sigma=\left(C_{1}, \ldots, C_{r}\right)$ in the chain complex of $\Delta_{\vec{c}}$ is defined by

$$
\partial\left(\left(C_{1}, \ldots, C_{r}\right)\right)=\sum_{i=1}^{r-1}(-1)^{i-1} \cdot\left(C_{1}, \ldots, C_{i} \cup C_{i+1}, \ldots, C_{r}\right)
$$

Lemma 5.2 For $\alpha \in \mathfrak{S}_{n}$, the element

$$
g_{\alpha}=\sum_{\gamma \in \mathfrak{S}_{\vec{c}}^{\prime}}(-1)^{\gamma} \cdot \sigma(\alpha \circ \gamma)
$$

in the chain group $C_{k-2}\left(\Delta_{\vec{c}}\right)$ belongs to the kernel of the boundary map and hence to the homology group $\widetilde{H}_{k-2}\left(\Delta_{\vec{c}}\right)$.

Theorem 5.3 The cycles $g_{\alpha}$, where $\alpha$ ranges over all permutations with descent composition $\vec{c}$, form a basis for the homology group $\widetilde{H}_{k-2}\left(\Delta_{\vec{c}}\right)$. 


\section{Representation of the symmetric group}

The symmetric group $\mathfrak{S}_{n}$ acts naturally on the the poset $\Pi_{\vec{c}}^{\bullet}$ by relabeling the elements. Hence it also acts on the order complex $\Delta\left(\Pi_{\vec{c}}^{\bullet}-\{\hat{1}\}\right)$. Lastly, the symmetric group acts on the top homology group $\widetilde{H}_{k-2}\left(\Delta\left(\Pi_{\vec{c}}^{\bullet}-\{\hat{1}\}\right)\right)$. We show in this section that this action is a Specht module of the border strip $B$ corresponding to the composition $\vec{c}$. For an overview on the representation theory of the symmetric group, we refer the reader to Sagan's book [12].

The forgetful map $\phi$ from $\operatorname{sd}\left(\Delta_{\vec{c}}\right)$ to the order complex of the poset $\Pi_{\vec{c}}^{\bullet}-\{\hat{1}\}$ commutes with the action of the symmetric group $\mathfrak{S}_{n}$. In other words, we have the commutative diagram

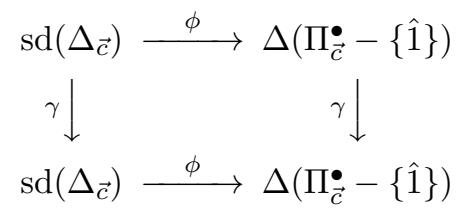

where $\gamma$ belongs to $\mathfrak{S}_{n}$. Observe that the map $\phi$ extends to a continuous function from the geometric realization $\left|\operatorname{sd}\left(\Delta_{\vec{c}}\right)\right|$ to the geometric realization $\left|\Delta\left(\Pi_{\vec{c}}^{\bullet}-\{\hat{1}\}\right)\right|$, and hence we have the map between the homology groups $\phi_{*}: \widetilde{H}_{k-2}\left(\operatorname{sd}\left(\Delta_{\vec{c}}\right)\right) \longrightarrow \widetilde{H}_{k-2}\left(\Delta\left(\Pi_{\vec{c}}^{\bullet}-\{\hat{1}\}\right)\right)$. By applying homology, we obtain that the following diagram of homology groups commutes:

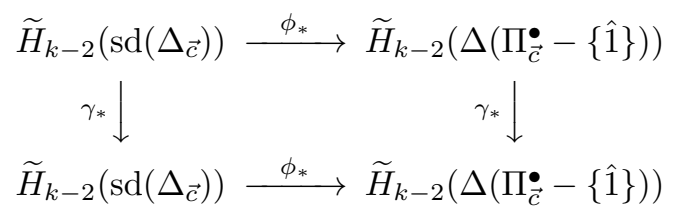

From the Quillen's fiber lemma we know that the function $\phi$ has a homotopic inverse, say $\psi: \mid \Delta\left(\Pi_{\vec{c}}^{\bullet}-\right.$ $\{\hat{1}\})|\longrightarrow| \operatorname{sd}\left(\Delta_{\vec{c}}\right) \mid$. Hence we have $\psi_{*}: \widetilde{H}_{k-2}\left(\Delta\left(\Pi_{\vec{c}}^{\bullet}-\{\hat{1}\}\right)\right) \longrightarrow \widetilde{H}_{k-2}\left(\operatorname{sd}\left(\Delta_{\vec{c}}\right)\right)$. Note that $\phi_{*}$ and $\psi_{*}$ are inverses of each other. The function $\psi$ may not commute with the group action. However, since $\gamma_{*} \circ \psi_{*}=\psi_{*} \circ \phi_{*} \circ \gamma_{*} \circ \psi_{*}=\psi_{*} \circ \gamma_{*} \circ \phi_{*} \circ \psi_{*}=\psi_{*} \circ \gamma_{*}$ we have that $\psi_{*}$ commutes with the group action. We formulate this statement as follows.

Proposition 6.1 The two homology groups $\widetilde{H}_{k-2}\left(\operatorname{sd}\left(\Delta_{\vec{c}}\right)\right)$ and $\widetilde{H}_{k-2}\left(\Delta\left(\Pi_{\vec{c}}^{\bullet}\{\hat{1}\}\right)\right)$ are isomorphic as $\mathfrak{S}_{n}$-modules.

It is clear that $\widetilde{H}_{k-2}\left(\operatorname{sd}\left(\Delta_{\vec{c}}\right)\right)$ and $\widetilde{H}_{k-2}\left(\Delta_{\vec{c}}\right)$ are isomorphic as $\mathfrak{S}_{n}$ modules. Hence in the remainder of this section we will study the action the symmetric group $\mathfrak{S}_{n}$ on $\Delta_{\vec{c}}$ and its action on the homology group $\widetilde{H}_{k-2}\left(\Delta_{\vec{c}}\right)$.

Let $B$ be the border strip that has $k$ rows where the $i$ th row consists of $c_{i}$ boxes. Recall that a tableau is a filling of the boxes of the shape $B$ with the integers 1 through $n$. A standard Young tableau is a tableau where the rows and columns are increasing. A tabloid is an equivalence class of tableaux under the relation of permuting the entries in each row. See [12, Section 2.1] for details.

Observe that there is a natural bijection between tabloids of shape $B$ and facets of the complex $\Delta_{\vec{c}}$ by letting the elements in each row form a block and letting the order of the blocks go from lowest to highest row. Let $M^{B}$ be the permutation module corresponding to shape $B$, that is, the linear span of all tabloids 
of shape $B$. Notice that the above bijection induces a $\mathfrak{S}_{n}$-module isomorphism between the permutation module $M^{B}$ and the chain group $C_{k-2}\left(\Delta_{\vec{c}}\right)$.

Furthermore, there is a bijection between tableaux of shape $B$ and and permutations by reading the elements in the northeast direction from the border strip. Recall that the group $\mathfrak{S}_{\vec{c}}^{\prime}=\mathfrak{S}_{K_{1}} \times \cdots \times \mathfrak{S}_{K_{n-k+1}}$ is the column stabilizer of the border strip $B$. Let $t$ be a tableau and $\alpha$ its association permutation. Hence the polytabloid $\mathbf{e}_{t}$ corresponding to the tableau $t$ is the element $g_{\alpha}$ presented in Lemma 5.2 Since the Specht module $S^{B}$ is the submodule of $M^{B}$ spanned by all polytabloids, Lemma 5.2 proves that the Specht module $S^{B}$ is isomorphic to a submodule of the kernel of the boundary map $\partial_{k-2}$. Since the kernel is the top homology group $\widetilde{H}_{k-2}\left(\Delta_{\vec{c}}\right)$, and the Specht module $S^{B}$ and the homology group $\widetilde{H}_{k-2}\left(\Delta_{\vec{c}}\right)$ have the same dimension $\beta(\vec{c})$, we conclude that they are isomorphic. To summarize we have:

Proposition 6.2 The top homology group $\widetilde{H}_{k-2}\left(\Delta_{\vec{c}}\right)$ is isomorphic to the Specht module $S^{B}$ as $\mathfrak{S}_{n^{-}}$ modules.

By combining Propositions 6.1 and 6.2, the main result of this section follows.

Theorem 6.3 The top homology group $\widetilde{H}_{k-2}\left(\Delta\left(\Pi_{\vec{c}}^{\bullet}-\{\hat{1}\}\right)\right)$ is isomorphic to the Specht module $S^{B}$ as $\mathfrak{S}_{n}$-modules.

\section{Knapsack partitions}

We now turn our attention to filters in the pointed partition lattice $\Pi_{n}^{\bullet}$ that are generated by a pointed knapsack partition. These filters were introduced in [4].

Recall that we view an integer partition $\lambda$ as a multiset of positive integers. Let $\lambda=\left\{\lambda_{1}^{e_{1}}, \ldots, \lambda_{q}^{e_{q}}\right\}$ be an integer partition, where we assume that the $\lambda_{i}$ 's are distinct. If all the $\left(e_{1}+1\right) \cdots\left(e_{q}+1\right)$ integer linear combinations

$$
\left\{\sum_{i=1}^{q} f_{i} \cdot \lambda_{i}: 0 \leq f_{i} \leq e_{i}\right\}
$$

are distinct, we call $\lambda$ a knapsack partition. A pointed integer partition $\{\lambda, \underline{m}\}$ is called a pointed knapsack partition if the partition $\lambda$ is a knapsack partition.

Definition 7.1 For a pointed knapsack partition $\{\lambda, \underline{m}\}=\left\{\lambda_{1}, \lambda_{2}, \ldots, \lambda_{k}, \underline{m}\right\}$ of $n$ define the subposet $\Pi_{\{\lambda, \underline{m}\}}^{\bullet}$ to be the filter of $\Pi_{n}^{\bullet}$ generated by all pointed set partitions of type $\{\lambda, \underline{m}\}$ and define the subcomplex $\Lambda_{\{\lambda, \underline{m}\}}$ of the complex $\Delta_{n}$ by

$$
\Lambda_{\{\lambda, \underline{m}\}}=\left\{\tau=\left(C_{1}, \ldots, C_{r-1}, C_{r}\right) \in \Delta_{n}:\left\{C_{1}, \ldots, C_{r-1}, \underline{C_{r}}\right\} \in \Pi_{\{\lambda, \underline{m}\}}^{\bullet}\right\} .
$$

For a pointed knapsack partition $\{\lambda, \underline{m}\}$ of $n$ define $F$ to be the filter in the poset of compositions of $n$ generated by compositions $\vec{c}$ such that type $(\vec{c})=\{\lambda, \underline{m}\}$. Now define $V(\lambda, \underline{m})$ to be the collection of all pointed compositions $\vec{c}=\left(c_{1}, c_{2}, \ldots, c_{r}\right)$ in the filter $F$ such that each $c_{i}, 1 \leq i \leq r-1$, is a sum of distinct parts of the partition $\lambda$ and $c_{r}=m$. As an example, for $\lambda=\{1,1,3,7\}$ we have $(4,8, m) \in V(\lambda, \underline{m})$ but $(2,10, m) \notin V(\lambda, \underline{m})$.

For a composition $\vec{d}$ in $V(\lambda, \underline{m})$ define $\epsilon(\vec{d})$ to be the composition of type $\{\lambda, \underline{m}\}$, where each entry $d_{i}$ of $\vec{d}$ has been replaced with a decreasing list of parts of $\lambda$. That is,

$$
\epsilon(\vec{d})=\left(\lambda_{1,1}, \ldots, \lambda_{1, t_{1}}, \ldots, \lambda_{s, 1}, \ldots, \lambda_{s, t_{s}}, m\right),
$$




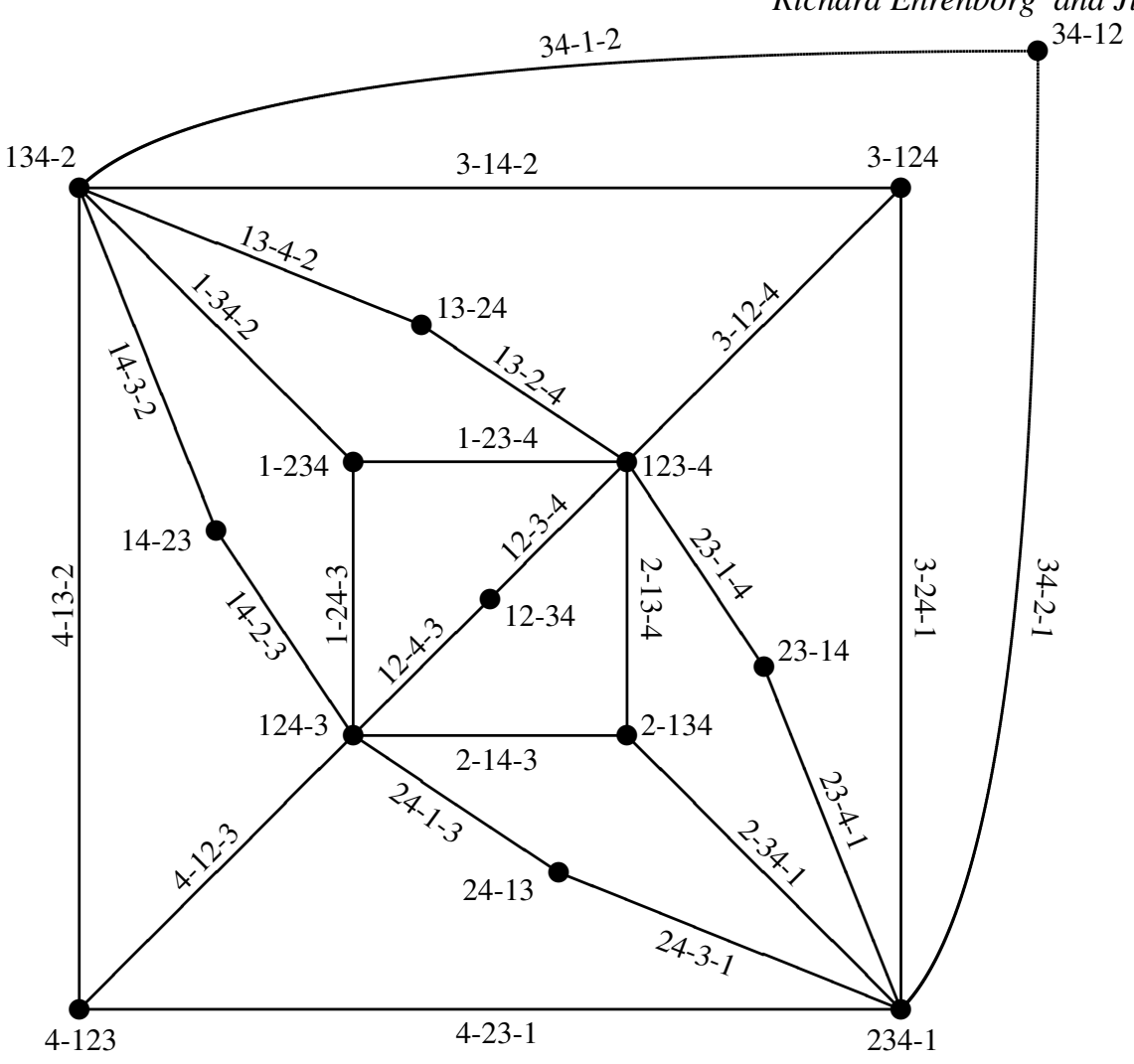

Fig. 2: The simplicial complex $\Lambda_{\{2,1, \underline{1}\}}$, corresponding to the knapsack partition $\{2,1, \underline{1}\}$. Notice that this complex is the union of two complexes $\Delta_{(1,2,1)}$ and $\Delta_{(2,1,1)}$.

where $\lambda_{i, 1}>\lambda_{i, 2}>\cdots>\lambda_{i, t_{i}}, \sum_{j=1}^{t_{i}} \lambda_{i, j}=d_{i}$ and

$$
\{\lambda, \underline{m}\}=\left\{\lambda_{1,1}, \ldots, \lambda_{1, t_{1}}, \ldots, \lambda_{s, 1}, \ldots, \lambda_{s, t_{s}}, \underline{m}\right\} .
$$

As an example, for the pointed knapsack partition $\lambda=\{2,1, \underline{1}\}$ we have $\epsilon((3,1))=(2,1,1), \epsilon((2,1,1))$ $=(2,1,1)$ and $\epsilon((1,2,1))=(1,2,1)$. Also note $\epsilon(\vec{d}) \leq \vec{d}$ in the partial order of compositions.

Similar to Theorem 3.2 we have the following topological conclusion. However, this time the tool is not shelling, but discrete Morse theory.

Theorem 7.2 There is a Morse matching on the simplicial complex $\Lambda_{\{\lambda, \underline{m}\}}$ such that the only critical cells are of the form $\sigma(\alpha, \epsilon(\vec{d}))$ where $\vec{d}$ ranges in the set $V(\lambda, \underline{m})$ and $\alpha$ ranges over all permutations in the symmetric group $\mathfrak{S}_{n}$ with descent composition $\vec{d}$. Hence, the simplicial complex $\Lambda_{\{\lambda, \underline{m}\}}$ is homotopy equivalent to wedge of $\sum_{\vec{d} \in V(\lambda, \underline{m})} \beta(\vec{d})$ spheres of dimension $k-1$.

Example 7.3 Consider the pointed knapsack partition $\{\lambda, \underline{m}\}=\{2,1, \underline{1}\}$, whose associated complex $\Lambda_{\{2,1, \underline{1}\}}$ is shown in Figure 2 Note that $V(\lambda, \underline{m})=\{(1,2,1),(2,1,1),(3,1)\}$. The critical cells of the 
complex $\Lambda_{\{2,1, \underline{1}\}}$ are as follows:

\begin{tabular}{c|c|c|l}
$\vec{d}$ & $\beta(\vec{d})$ & $\epsilon(\vec{d})$ & critical cells \\
\hline$(1,2,1)$ & 5 & $(1,2,1)$ & $2-14-3,3-14-2,3-24-1,4-13-2,4-23-1$ \\
$(2,1,1)$ & 3 & $(2,1,1)$ & $14-3-2,24-3-1,34-2-1$ \\
$(3,1)$ & 3 & $(2,1,1)$ & $12-4-3,13-4-2,23-4-1$
\end{tabular}

Note that $\Lambda_{\{2,1, \underline{1}\}}$ is homotopy equivalent to a wedge of 11 circles.

Now by the same reasoning as in Section 4 that is, using the forgetful map $\phi$ and Quillen's fiber lemma, we obtain the homotopy equivalence between the order complex of pointed partitions $\Pi_{\{\lambda, m\}}^{\bullet}-\{\hat{1}\}$ and the simplicial complex of ordered set partitions $\Lambda_{\{\lambda, \underline{m}\}}$.

Theorem 7.4 The order complex $\Delta\left(\Pi_{\{\lambda, \underline{m}\}}^{\bullet}-\{\hat{1}\}\right)$ is homotopy equivalent to the barycentric subdivision $\operatorname{sd}\left(\Lambda_{\{\lambda, \underline{m}\}}\right)$ and hence the simplicial complex $\Lambda_{\{\lambda, \underline{m}\}}$.

As a corollary we obtain the Möbius function of the poset $\Pi_{\{\lambda, \underline{m}\}}^{\bullet} \cup\{\hat{0}\}$; see [4].

Corollary 7.5 (Ehrenborg-Readdy) The Möbius function of the poset $\Pi_{\{\lambda, \underline{m}\}}^{\bullet} \cup\{\hat{0}\}$ is given by

$$
\mu\left(\Pi_{\{\lambda, \underline{m}\}}^{\bullet} \cup\{\hat{0}\}\right)=(-1)^{k} \cdot \sum_{\vec{d} \in V(\lambda, \underline{m})} \beta(\vec{d}) .
$$

By the same reasoning as Sections 5 and 6 , we have the following isomorphism.

Theorem 7.6 The two homology groups $\widetilde{H}_{k-1}\left(\Delta\left(\Pi_{\{\lambda, \underline{m}\}}^{\bullet}-\{\hat{1}\}\right)\right)$ and $\widetilde{H}_{k-1}\left(\Lambda_{\{\lambda, \underline{m}\}}\right)$ are isomorphic as $\mathfrak{S}_{n}$-modules. Furthermore, they are isomorphic to the direct sum of Specht modules

$$
\bigoplus_{\vec{d} \in V(\lambda, \underline{m})} S^{B(\vec{d})}
$$

\section{Concluding remarks}

We have not dealt with the question whether the poset $\Pi_{\vec{c}}^{\bullet}$ is $E L$-shellable. Recall that Wachs proved that the $d$-divisible partition lattice $\Pi_{n}^{d} \cup\{\hat{0}\}$ has an $E L$-labeling. Ehrenborg and Readdy gave an extension of this labeling to prove that $\Pi_{(d, \ldots, d, m)}^{\bullet}$ is $E L$-shellable [5]. Furthermore, Woodroofe [20] showed that the order complex $\Delta\left(\Pi_{n}^{d}-\{\hat{1}\}\right)$ has a convex ear decomposition. This is not true in general for $\Delta\left(\Pi_{\vec{c}}^{\bullet}-\{\hat{1}\}\right)$.

Can these techniques be used for studying other subposets of the partition lattice? One such subposet is the odd partition lattice, that is, the collection of all partition where each block size is odd. More generally, what can be said about the case when all the block sizes are congruent to $r$ modulo $d$ ? These posets have been studied in [3] and [18]. Moreover, what can be said about the poset $\Pi_{\{\lambda, \underline{m}\}}^{\bullet}$ when $\{\lambda, \underline{m}\}$ is not a pointed knapsack partition?

Another analogue of the partition lattice is the Dowling lattice. Subposets of the Dowling lattice have been studied in [5] and [8,9]. Here the first question to ask is what is the right analogue of the ordered set partitions. 


\section{Acknowledgements}

The authors thank Serge Ochanine for many helpful discussions and Margaret Readdy for her comments on an earlier draft of this paper.

\section{References}

[1] A. BJÖRnER, Shellable and Cohen-Macaulay partially ordered sets, Trans. Amer. Math. Soc. 260 (1980), 159183.

[2] A. BJÖRNER, Topological methods in Handbook of combinatorics, Vol. 1, 2, 1819-1872, Elsevier, Amsterdam, 1995.

[3] A. R. Calderbank, P. Hanlon and R. W. Robinson, Partitions into even and odd block size and some unusual characters of the symmetric groups, Proc. London Math. Soc. (3) 53 (1986), 288-320.

[4] R. Ehrenborg And M. Readdy, The Möbius function of partitions with restricted block sizes, Adv. in Appl. Math. 39 (2007), 283-292.

[5] R. Ehrenborg And M. Readdy, Exponential Dowling structures, European J. Combin. 30 (2009), 311-326.

[6] R. Forman, Morse theory for cell complexes, Adv. Math. 134 (1998), 90-145.

[7] R. Forman, A user's guide to discrete Morse theory, Sém. Lothar. Combin. 48 (2002), Article B48c.

[8] E. Gottlieb, On the homology of the $h, k$-equal Dowling lattice, SIAM J. Discrete Math. 17 (2003), 50-71.

[9] E. Gottlieb And M. L. Wachs, Cohomology of Dowling lattices and Lie (super)algebras, Adv. Appl. Math. 24 (2000), 301-336.

[10] D. KozLov, “Combinatorial Algebraic Topology,” Springer, 2008.

[11] D. Quillen, Homotopy properties of the poset of nontrivial p-subgroups of a group, Adv. Math. 28 (1978), 101-128.

[12] B. E. SAGAN, “The Symmetric Group, Second Edition,” Springer, 2000.

[13] R. P. Stanley, Exponential structures, Stud. Appl. Math. 59 (1978), 73-82.

[14] R. P. Stanley, "Enumerative Combinatorics, Vol. I,” Wadsworth and Brooks/Cole, Pacific Grove, 1986.

[15] R. P. StAnLEY, “Enumerative Combinatorics, Vol. II,” Cambridge University Press, 1999.

[16] G. S. Sylvester, “Continuous-Spin Ising Ferromagnets,” Doctoral dissertation, Massachusetts Institute of Technology, 1976.

[17] M. L. WACHS, A basis for the homology of the $d$-divisible partition lattice, Adv. Math. 117 (1996), $294-318$.

[18] M. L. WACHS, Whitney homology of semipure shellable posets, J. Algebraic Combin. 9 (1999), 173-207.

[19] J. W. WALKER, Homotopy type and Euler characteristic of partially ordered sets, European J. Combin. 2 (1981), 373-384.

[20] R. Woodroofe, Cubical convex ear decompositions, Electron. J. Combin. 16 (2009), 33 pp. 